\title{
Análise do impacto do Turnaround Time de reparáveis na disponibilidade da frota do Projeto C-95M
}

\author{
Mariane Camargos Figueiredo ${ }^{1}$ \\ Leila Scanfone ${ }^{2}$ \\ Daniel Coelho Mota ${ }^{3}$
}

\section{Resumo}

Este trabalho analisa a relação entre Turnaroud Time (TAT) médio de reparáveis e o impacto na disponibilidade da frota do Projeto C-95M, utilizando dados históricos da Força Aérea Brasileira (FAB). Tal abordagem é de suma importância para o trabalho desenvolvido na organização, pois os resultados podem impactar na necessidade do aumento de giro disponível, o que acarreta maior quantidade orçamentária e/ou menor quantidade de aeronaves prontas para voo. O objetivo deste trabalho é levantar o impacto do TAT na disponibilidade das aeronaves e identificar parâmetros para o TAT que possibilitem a melhoria no processo de aquisições ou contratação de serviços. Este intento é conseguido através de revisão bibliográfica a respeito do assunto, levantamento de dados da realidade da Força Aérea Brasileira e utilização do OPUS 10 na etapa de modelagem para verificação dos resultados. A análise demonstrou que o impacto do TAT na disponibilidade é maior quando a quantidade de giro é menor. Para o acervo do Projeto C-95M da FAB, foi possível verificar que o impacto é acentuado quando o TAT ultrapassa 120 dias, aproximadamente. Dessa forma, foi possível identificar que esse parâmetro é vantajoso na utilização em novas aquisições ou contratação de serviços.

Palavras-chave: TAT. Disponibilidade. C-95M. Força Aérea Brasileira.

\footnotetext{
${ }^{1}$ Especialista em Logística pelo Instituto de Logística da Aeronáutica, especialista em Gestão de Projetos pela Fundação Dom Cabral e bacharel em Engenharia Industrial Elétrica pelo Centro Federal de Educação Tecnológica de Minas Gerais. E-mail: mcamargos.figueiredo@gmail.com.

${ }^{2}$ Doutora em Administração pela Universidade Federal de Minas Gerais e mestre e bacharel em Administração pela Pontifícia Universidade Católica de Minas Gerais. E-mail: scanfone@unis.edu.br.

${ }^{3}$ Mestre em Pesquisa Operacional Militar pela Cranfield University, especialista em Logística pelo Instituto de Logística da Aeronáutica e bacharel em Engenharia Mecânica-Aeronáutica pelo Instituto Tecnológico de Aeronáutica. E-mail: danieldem3@fab.mil.br.
} 


\section{Introdução}

Entre os recursos destinados aos sistemas aeronáuticos, grande parte é consumida com os itens reparáveis. Esses, que possuem a característica de obter o custo de conserto e reuso mais atrativo que o descarte, tipicamente apresentam alto valor de aquisição e elevado tempo de vida (JR. e SRIVASTAVA, 1997). Segundo Sherbrooke (2004), apesar desses itens contarem, geralmente, com uma demanda igual a um, é necessária atenção no gerenciamento, pois, por se tratarem de itens complexos, compreendem a maior parte do orçamento e tendem a ter elevado tempo de reparo. Dessa forma, especificamente na aviação, que contempla numerosos e complexos sistemas, os desafios do gerenciamento de previsão de demanda e de estoques dificultam a tomada de decisão. Ainda quanto aos reparáveis, esses podem ser classificados de duas formas: Line Replaceable Unit (LRU), que no caso de falha podem ser substituídos diretamente no terminal, ou Shop Replaceable Unit (SRU), que não é possível a troca direta na linha. Dessa forma, no caso de falha da SRU, é necessária a retirada da LRU mãe daquele componente para envio para reparo em uma oficina (WU e HSU, 2008).

Devido ao elevado número de aeronaves e projetos atendidos pela $\mathrm{FAB}$, aliados ao limite orçamentário, é necessário que as decisões sejam tomadas, preferencialmente, com vistas ao processo como um todo. Alinhado a esse conceito, cita-se Sherbrooke (2004), que descreve a abordagem por item, utilizada por anos, focada na decisão individual, ou seja, na definição de quantidade de estoque a se comprar e que apresentou desvantagens em relação à abordagem sistêmica. Nesta, tem-se maior controle dos resultados de interesse e a possibilidade de traçar uma curva custo-eficiência para o todo.

As variáveis de controle, disponibilidade e custo, estão intimamente relacionadas com o prazo de suprimento dos itens, que pode ser definido como o tempo entre um equipamento apresentar uma falha em uma aeronave até a disponibilidade do mesmo ou outro igual para instalação no avião. Assim, no caso de falha, há a possibilidade de existir uma peça sobressalente em estoque para instalação (aos itens disponíveis, além das unidades instaladas, dá-se o nome de giro). Como também há o caso da não existência de estoque e o item em pane deverá ser retirado e encaminhado para reparo, que poderá ocorrer na base, no parque ou em empresa contratada. O tempo entre a desinstalação até a disponibilidade do item reparado para uso é definido como o turnaround time.

A aplicação de um estudo que estima a quantidade demandada e a distribuição dos itens é importante para identificar melhorias e erros que ocorrem na rotina de planejamento dos equipamentos, como identificação de oportunidades nos processos de contratação (certeza 
maior do TAT e da quantidade de itens), aquisições realizadas com maior precisão dos equipamentos, entre outros. A utilização de um software para a análise do problema é necessário, pois o elevado número de sistemas, com diferentes níveis de manutenção, com hierarquia dos itens, torna o desafio complexo sem uma ferramenta computacional. E, ainda, o software possibilita agilizar o processo de análise, fator importante para o dinamismo do mundo atual.

O C-95M (EMBRAER 110) está inserido na frota de aeronaves de transporte da Força Aérea Brasileira, que contempla em seu acervo 46 aeronaves distribuídas entre 12 operadores. Com a idade média da frota de aproximadamente 35 anos, o projeto passou, a partir de 2008, por um processo de modernização dos sistemas aviônicos, o que acarretou na extensão do seu ciclo de vida até 2029. De acordo com o as informações de planejamento disponíveis no Sistema Integrado de Logística de Material e Serviços (SILOMS), a frota apresentou um esforço aéreo previsto para 2018 de 17.592 horas, o que representa cerca de $15 \%$ do esforço da FAB e, no planejamento de recursos financeiros anuais, consome cerca de $25 \%$ da previsão do Parque de Material Aeronáutico de Lagoa Santa (PAMA-LS) - responsável por esse projeto e mais outros oito.

Este trabalho analisa a aplicação da abordagem sistêmica em um modelo de múltiplo escalão para manutenção de reparáveis LRU da aeronave C-95M da Força Aérea Brasileira. Foi realizado um detalhamento de como o TAT impacta na disponibilidade, fator determinante de performance da frota, e identifica parâmetros de melhoria do processo de aquisição e contratação. O modelo adotado pela FAB é de dois escalões, em que o Parque Central do projeto atende todos os operadores do Brasil. Tal abordagem se faz necessária, pois a estratégia de manutenção da aeronave C-95M é realizada por meio de vários contratos para os diversos sistemas e o TAT é um parâmetro que interfere nos resultados de disponibilidade e na definição de giro. É importante ressaltar também a contribuição do trabalho para a Força Aérea, quando poderá auxiliar em futuras contratações ou identificar falhas de planejamento.

\section{Referencial Teórico}

A definição da quantidade de estoques de reparáveis é mais complexa que a de materiais de consumo, pois há decisões que devem ser tomadas, como a taxa de condenação para definir o momento de novas aquisições e qual o tipo de manutenção deverá ser realizado no equipamento. Ademais, pode ser complicada por outros fatores, como o retorno do reparo não 
ser cadenciado com a demanda, o que irá influenciar na metodologia de retorno do item (JR. e SRIVASTAVA, 1997).

Em Mota (2014), o autor afirma que os componentes de sistemas complexos, como o de aeronaves, são construídos para que, no caso de falha, possam ser substituídos para reparo fora do local de operação. E o gerenciamento dos itens de reparo encontra desafios nas restrições orçamentárias, demanda esporádica e instável, longos tempos de manutenção, capacidade limitada para reparar, entre outros.

Um sistema de inventário de reparáveis tem, geralmente, uma estrutura hierárquica em que o reparo é realizado em uma sede central, em que há locais de armazenamento, e o cliente aguarda o envio de uma peça sobressalente. Essa organização tem o objetivo de fornecer respostas rápidas de suprimento e redução de custos (MOTA, 2014).

Lau e Song (2004) citam que, para tratar esse tipo de estrutura, o Multi-Echelon Technique for Recoverable Item Control (METRIC) é o método pioneiro apresentado por Sherbrooke (2004), baseado num processo de Poisson que utiliza uma análise marginal de custo e disponibilidade, como forma de otimização.

\subsection{O modelo dos Múltiplos Escalões}

O modelo de múltiplos escalões trabalha com a seguinte metodologia: no caso da falha em um item, ele é retirado da aeronave e verificado o reparo na base, enquanto isso, é solicitado um sobressalente para o próprio estoque. Se existir o item no estoque da base, ele será atendido e a aeronave ficará disponível. Caso contrário, a existência do pedido de material representará a quantidade de itens faltantes na frota e, a essa ordem em aberto, dá-se o nome de Backorder (BO). O pedido só será atendido no caso da chegada do mesmo no estoque, seja por reparo na própria base - caso isso seja possível, após o item ter sido reparado em lugar específico para isso ou da entrega de outro, enviado do estoque central.

Dessa forma, o modelo de múltiplos escalões é capaz de calcular o nível de estoque ótimo nas bases e armazém, para cada item. O BO na base que irá causar a indisponibilidade e, assim, o objetivo do modelo é obter o menor valor esperado dessas ordens, ou seja, minimizar o Expected Backorder (EBO) (SHERBROOKE, 2004). Em Mota (2014), são detalhados o Multi-Eschelon Technique for Recoverable Item Control (METRIC) e o VARI-METRIC. Basicamente, esses métodos utilizam a técnica do múltiplo escalão detalhado, sendo que o VARI-METRIC é um modelo de segunda ordem que difere do primeiro pelo cálculo do EBO, pois incorpora dois parâmetros: média e variância, aprimorando o seu cálculo. 
O parâmetro do turnaround time é significativo nesse tipo de organização e é definido em Sherbrooke (2004) como tempo de ordem e de entrega, ou seja, descreve o tempo que leva entre a abertura do pedido de determinado item que é retirado para reparo até o momento do recebimento do mesmo item do armazém.

Em Graves (1985), o autor cita que no meio militar essa técnica é bem utilizada e afirma que o sistema múltiplo escalão, em sua forma mais simples, é composto por um local de reparo central que suporta uma quantidade de operadores. Em seu trabalho de dois escalões, assumiu que em todos centros de operação a falha consistia de um processo de Poisson, com média constante, independente dos itens em funcionamento e que o tempo de reparo é determinístico para cada item e possuem o mesmo valor. Em Lau e Song (2004), os autores abordam o contexto militar para otimização de alocação de sobressalente num modelo de dois escalões com demanda variável e capacidade de reparo limitada, assim, eles utilizam a metodologia de filas para o sistema gerar os resultados de análise marginal e EBO.

Os autores JR. e Srivastava (1997) descrevem o problema típico da logística militar no gerenciamento de reparáveis para as aeronaves, em que as bases são capazes de fazer algumas manutenções e que outras são centralizadas em um local que suportam todos os locais de operação do item. E, assim, a falta de componentes em uma aeronave a tornará indisponível e o objetivo do sistema consta na maximização da disponibilidade sujeito a restrições orçamentárias.

\subsection{A Subcontratação no Mercado Competitivo}

Segundo Freitas (2014), as empresas de manufatura e varejo têm um crescimento intenso na demanda de terceirização das funções logísticas, devido à competição intensificada na economia emergente global, como forma efetiva de redução de custos e compartilhamento de riscos. A escolha de subcontratar ou quais as estratégias utilizar é um desafio para todo gestor e irá impactar na performance geral da empresa. Se, por um lado, a terceirização leva as vantagens de passar o processo para uma empresa especializada, em um processo no qual a contratante passa a ser cliente; por outro lado, a organização perde o seu conhecimento técnico especializado e passa a depender dos serviços de terceiros. Além disso, há a necessidade de um aperfeiçoamento dos processos, pois é necessário que os contratos sejam bons para ambos os lados e que as empresas se tornem parceiras para um resultado mais eficaz. Com isso, riscos e penalidades são fatores que devem ser bem definidos, pois vão contra os princípios de parceria. 
Os autores Waak e Sturgess (2000) descrevem, em seu trabalho, os riscos na terceirização do suporte e descrevem técnicas de determinação de um contrato de suporte logístico. No artigo, é detalhado que o processo de suporte deve ser iterativo com o processo de engenharia; para novos sistemas, onde o risco aumenta pelo desconhecimento, é necessária a geração de dados e também a definição de uma baseline que, através do acompanhamento desses processos, torne possível a avaliação do sistema como um todo.

Sleiderink (2015) afirma que há uma tendência na contratação de terceiros por disponibilidade (suporte por serviço) e capacidade (cliente não tem a propriedade do sistema) e se faz com o intuito de redução de custos e aumento de eficiência. Entre os motivos destacados, estão: a falta de conhecimento e de recursos do cliente. O trabalho aborda os contratos do tipo Performance Based Logistics (PBL) na entrega de disponibilidade, através da análise do impacto de penalidade e da avaliação de estoque de itens de entrega rápida e lenta, de localização de estoque e da alteração do período de revisão. Por meio de simulações, o autor aponta que os custos de penalidade são reduzidos em função do investimento em itens de entrega lenta. Além disto, o aumento do tempo de reparo dos itens é mais viável, e há melhor utilização de recursos para redução do tempo de reabastecimento do que para a diminuição da taxa de falha.

\subsection{OPUS 10}

O OPUS 10 é um programa da empresa Systecon que é utilizado para otimização logística, seja através de análise de estoque ou de suporte. O METRIC e o VARI-METRIC são as bases matemáticas do seu modelo. Assim, é um modelo matemático que usa os dados informados pelo operador e, portanto, não é um software de simulação de eventos discretos (MOTA, 2014). O software é amplamente utilizado em grandes empresas para análise de decisão sistêmica. Entre suas aplicações, destacam-se análise da lista de aprovisionamento inicial (LAI), estudo de estoque (rearranjo, recompletamento - ou ambos), avaliação de estrutura de suporte logística e análise de sensibilidade. Na literatura, há diversas aplicações dessa ferramenta no âmbito gerencial.

Em Wu e Hsu (2008), os autores propõem uma definição de uma lista de materiais de compra de peças de reposição através da análise dos vendedores possíveis para os diversos itens, considerando a taxa de falha e custo específico de cada fornecedor/item. No trabalho, é analisado, além do custo, a taxa de falha que irá impactar na quantidade de estoque necessária do sistema e que varia de acordo com o vendedor. Devido à elevada quantidade 
item/fornecedor, o OPUS 10 levaria bastante tempo para encontrar a solução ótima para o sistema. Assim, os autores utilizaram as fórmulas matemáticas de cálculo de disponibilidade do OPUS para gerar um algoritmo genético de busca de solução ótima.

Tysseland (2009) aborda a otimização de sobressalentes no sistema de defesa da Noruega utilizando o OPUS 10 e discute se os resultados encontrados se sustentam como econômicos. A escolha desse software se deu após uma análise de estoque que indicou investimentos em giro nunca utilizados. Apesar de centenas de projetos do órgão, o software foi utilizado em apenas alguns, devido à falta de dados, e evidenciou o grande desafio encontrado na falta de pessoal qualificado em gerenciamento de estoque. Uma constatação apresentada pelo autor é que os fornecedores, quando vão produzir a lista inicial de sobressalentes, não a fazem baseados em Mean Time Between Fail (MTBF) e TAT - que são representativos da disponibilidade, mas focam na maior quantidade de peças a serem vendidas e, ainda, verificou-se que os projetos que possibilitaram a avaliação, após aplicação do OPUS, revelaram uma redução média de custos de $60 \%$ (sessenta por cento).

No trabalho de Wjk e Andersson (2012), é realizada uma análise dos contratos Performance Based Logistics (PBL), que basicamente consistem na contratação de empresas para a entrega de uma performance sujeita à aplicação de incentivos e penalidades. O cenário do estudo apresentou um sistema de três escalões, em que o fornecedor é responsável por suportar (com estoque e manutenção) a disponibilidade em um certo percentual e, como métrica de performance, o EBO foi utilizado. A partir do estoque sugerido pelo OPUS, a ferramenta SIMLOX é utilizada para verificar a variação no número de EBO, dado importante para definição da função de penalidade e, assim, é possível a avaliação de cliente e vendedor quanto à aplicabilidade do contrato e riscos envolvidos.

\section{Materiais e Métodos}

O presente estudo visa identificar o impacto do TAT na disponibilidade da frota das aeronaves C-95M da FAB e propor melhorias aos processos existentes. Para tal fim, foi realizada uma pesquisa aplicada, de campo, quantitativa e exploratória, na qual foram levantados dados de trinta e quatro itens de manutenção distintos e reparáveis, os quais foram aplicados no software OPUS 10 para verificação e análise de resultados. Já o levantamento dos dados foi realizado por meio do Sistema Integrado de Logística de Material e Serviços (SILOMS), que consiste no sistema empregado na Força Aérea. 


\subsection{Levantamento de Dados}

Para análise do impacto do TAT na disponibilidade da frota, foi realizado um levantamento de dados de dez anos de Ordem de Serviço (OS), ocorridas entre os períodos de janeiro de 2008 a dezembro de 2017, no Sistema Integrado de Logística de Material e Serviços (SILOMS), software de gerenciamento de manutenção e estoque que a FAB utiliza para todas as suas unidades e equipamentos. Entretanto, esse sistema apresenta diversos erros de lançamento humano e, por isso, a necessidade de tratamento das informações. Para limpeza dos dados que trariam informações errôneas, foram desconsideradas as OS`s que não apresentaram informações de unidade solicitante, nem data fim real ou data fim anterior à inicial, quantidades enviadas para manutenção maior do que um (hábito comum de operadores para acerto de estoque no SILOMS), itens que foram recolhidos apenas uma vez e Part Numbers (PN) sem dados de Mean Time Between Removal (MTBR). E, de um total de sessenta e cinco mil dados, após a limpeza, o banco de informações passou para um valor aproximado de vinte e um mil, constando de noventa e oito PN`s diferentes.

Em um primeiro momento, os valores de MTBR apresentaram números muito elevados, ocasionando erros no OPUS. Diante disso, foi gerado o relatório de todas as Fichas de Coletas de Dados de Defeitos (FCDD) para o projeto dos últimos dez anos, conforme havia sido realizado para os outros dados. Com os dados das FCDD`s encontrados e tratados, a quantidade de PN`s analisados diminuiu para trinta e quatro.

\subsection{Aplicações Matemáticas}

A disponibilidade operacional do sistema representa o percentual de aeronaves disponíveis para voo, descontando-se os tempos de manutenção e atrasos de suprimentos. Matematicamente, ela é definida pela seguinte expressão (MOTA, 2014):

Disponibilidade Operacional $=\frac{\mathrm{MTBM}}{\mathrm{MTBM}+\mathrm{MCMT}+\mathrm{MPMT}+\mathrm{MSD}}$

Onde:

MTBM (Mean Time Between Maintenance) é o tempo médio entre as manutenções;

MCMT (Mean Corrective Maintenance Time) tempo médio que o item leva em manutenção corretiva; 
MPMT (Mean Preventive Maintenance Time) tempo médio que o item fica em manutenção preventiva; e

MSD (Mean Supply Delay) tempo médio de suprimento do item.

No presente estudo, não foram descontados os tempos de indisponibilidades por manutenção preventiva. Assim, o foco da análise se concentra na disponibilidade por suprimento, que compreende na análise dos parâmetros de MTBM (que no estudo é constante) e MSD que engloba os tempos de transporte, reparo e entrega, desde a falha até a disposição do item para uso, portanto, incorpora o TAT. Variando este dado, o tempo por suprimento será alterado e irá afetar o EBO. Quanto maior o tempo para retorno de um item para uso, maior o número de backorders, logo, maior o giro necessário para atendimento da frota.

A disponibilidade por suprimento é calculada pela seguinte expressão (SHERBROOKE, 2004):

Disponibilidade por Suprimento $=\frac{100 \times \mathrm{MTBM}}{\mathrm{MTBM}+\mathrm{MSD}}$

No modelo, é assumido que a demanda segue uma distribuição de Poisson com média constante, e a escolha deu-se pelo modelo apresentar boa resposta para demandas aleatórias.

A taxa de falha dos equipamentos foi calculada através da seguinte expressão, onde o mean time between fail (MTBF) foi retirado dos dados das FCDD`s:

$$
\lambda=\frac{1}{M T B F}
$$

Onde $\lambda$ representa a taxa de falha.

Para o estudo em caso:

$$
\frac{1}{M T B M_{A N V}}=\sum \lambda_{I T E N S}
$$

MTBM $_{\text {ANv }}$ é o tempo médio entre as manutenções das aeronaves.

\section{Apresentação de Dados e Resultados}

Os sistemas de manutenção das frotas de aeronaves da FAB são realizados através dos modelos de múltiplo escalão. Assim, no caso específico das aeronaves C-95, o PAMA-LS é responsável por planejar toda a aquisição de materiais, diagonal de manutenção da frota e fazer toda a interface com as contratadas e o fabricante. Em alguns casos de contratações e análise de estoque, o Parque pede apoio ao Instituto Logístico da Aeronáutica (ILA) para o assessoramento, que é feito através do auxílio do software OPUS10. 
Em sua maioria, o planejamento de contratação de manutenção de reparáveis é realizado através da utilização de dados históricos, ou seja, por meio da análise das médias anuais de necessidades ocorridas durante os anos anteriores. Quanto à localização do estoque, não há um estudo detalhado sobre onde estarão estocados os equipamentos. Grande parte encontra-se localizado no estoque central, outra no operador com o maior número de aeronaves e as outras são distribuídas para organizações mais distantes, como na região Norte do país, e outros têm a quantidade zerada.

\subsection{OPUS 10}

O estudo consta de um problema de dois escalões, estrutura aplicada na Força Aérea, compostos por itens reparáveis apenas do tipo LRU. Desta forma, no caso de falha em um item, ele será retirado da aeronave e uma ordem de serviço para manutenção da unidade será registrada. Caso haja estoque no operador, outra unidade em funcionamento será colocada no lugar; caso contrário, o operador possuirá uma ordem de serviço em aberto na própria base e a aeronave ficará indisponível até a chegada de outro para suprimento. O item será enviado para reparo no Parque central. No sistema simulado, não há condenações, nem canibalização, os itens não são ressupridos lateralmente e não é considerada a existência de filas na manutenção, ou seja, a capacidade de reparo é ilimitada.

A organização do modelo está representada na figura 01, em que o PAMALS teve a sua estação classificada como DEPOT, ou seja, local onde os itens são reparados e estocados, e os operadores AFA, EEAR, GEIV, IPEV, PAMASP, ALA 1, ALA 3, ALA 9, ALA 10, ALA 12 e ALA15, foram classificadas como STORE - apenas permitem a estocagem dos itens. Assim, são quarenta e cinco sistemas C-95M distribuídos em 12 organizações militares, sendo uma destas o próprio parque central, que também é operador da aeronave. 
Figura 01 - Organização do Suporte Logístico.

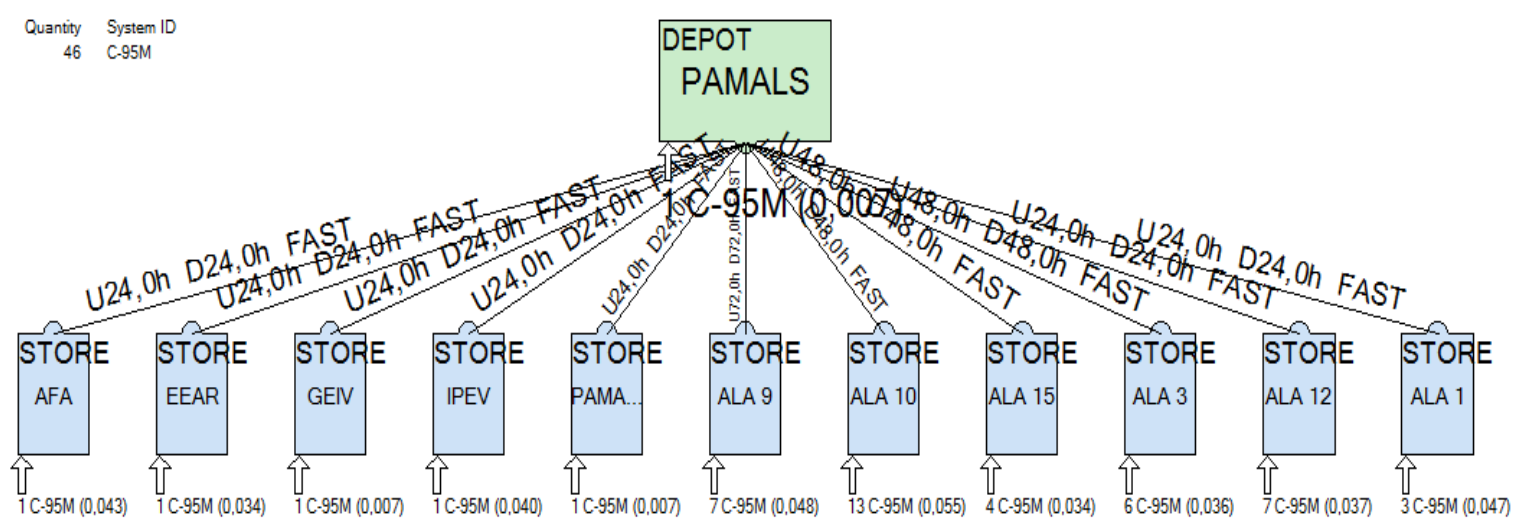

Fonte: os autores.

Dentre as ferramentas de análise disponíveis no OPUS, foi escolhida a análise de ressuprimento e rearranjo, que fornece resultados de melhoria nos processos logísticos, através da indicação de quais itens devem seguir a preferência de compra e como deve ser distribuído o estoque nos vários locais possíveis. Para cada sistema C-95M, foram considerados trinta e quatro reparáveis dos diversos subsistemas da aeronave: motor, trem de pouso, partes eletrônicas, etc. Os dados considerados (custo, quantidade por aeronave (QPA), TAT e taxa de falha) foram extraídos do SILOMS e estão detalhados na tabela 01.

Tabela 01 - Tabela de dados aplicados no OPUS 10.

(continua)

\begin{tabular}{|c|c|c|c|c|c|}
\hline PN & QPA & CUSTO (US\$) & TAT MÉDIO (H) & TAT MÉDIO (DIAS) & TX FALHA \\
\hline ITEM 1 & 1 & $7.565,00$ & 13646,74 & 98 & 2,02 \\
\hline ITEM 2 & 1 & 952,98 & 8810,94 & 367 & 227,44 \\
\hline ITEM 3 & 1 & $184.724,30$ & 2622,01 & 303 & 16,91 \\
\hline ITEM 4 & 1 & $132.079,19$ & 4944,00 & 118 & 37,84 \\
\hline ITEM 5 & 1 & $2.780,75$ & 1756,80 & 253 & 73,55 \\
\hline ITEM 6 & 1 & $87.917,33$ & 9394,29 & 234 & 251,57 \\
\hline ITEM 7 & 3 & $1.400,00$ & 6484,80 & 270 & 11,13 \\
\hline ITEM 8 & 2 & $2.585,26$ & 4872,00 & 100 & 646,83 \\
\hline ITEM 9 & 2 & $9.155,74$ & 3209,23 & 140 & 939,88 \\
\hline
\end{tabular}


Tabela 01 - Tabela de dados aplicados no OPUS 10.

(conclusão)

\begin{tabular}{|c|c|c|c|c|c|}
\hline PN & QPA & CUSTO (US\$) & TAT MÉDIO (H) & TAT MÉDIO (DIAS) & TX FALHA \\
\hline ITEM 10 & 1 & $10.781,25$ & 3304,32 & 660 & 10,19 \\
\hline ITEM 11 & 1 & $12.581,04$ & 516,84 & 564 & 382,62 \\
\hline ITEM 12 & 4 & $1.648,35$ & 3351,38 & 203 & 157,16 \\
\hline ITEM 13 & 1 & $7.777,77$ & 6324,38 & 58 & 1024,47 \\
\hline ITEM 14 & 1 & $4.074,76$ & 8155,65 & 73 & 1283,33 \\
\hline ITEM 15 & 2 & $3.333,33$ & 3089,98 & 129 & 2,07 \\
\hline ITEM 16 & 1 & $4.215,34$ & 7368,30 & 36 & 572,07 \\
\hline ITEM 17 & 1 & $2.685,00$ & 1392,00 & 340 & 1,54 \\
\hline ITEM 18 & 1 & $2.184,62$ & 13536,00 & 307 & 13,88 \\
\hline ITEM 19 & 1 & $1.000,00$ & 7262,40 & 72 & 558,03 \\
\hline ITEM 20 & 2 & $1.284,14$ & 5613,33 & 77 & 10000 \\
\hline ITEM 21 & 2 & $12.750,00$ & 2913,33 & 159 & 5,50 \\
\hline ITEM 22 & 2 & $37.688,13$ & 2802,00 & 569 & 1,51 \\
\hline ITEM 23 & 1 & $6.500,00$ & 3818,04 & 264 & 1,29 \\
\hline ITEM 24 & 2 & $2.000,00$ & 15835,20 & 321 & 0,85 \\
\hline ITEM 25 & 1 & $7.990,00$ & 7709,65 & 133 & 2914,23 \\
\hline ITEM 26 & 1 & $8.898,00$ & 3201,88 & 134 & 388,64 \\
\hline ITEM 27 & 2 & $1.572,08$ & 2411,08 & 138 & 7633,59 \\
\hline ITEM 28 & 2 & $4.585,00$ & 1724,76 & 22 & 909,09 \\
\hline ITEM 29 & 1 & $1.013,83$ & 2829,37 & 121 & 25,01 \\
\hline ITEM 30 & 2 & $3.391,00$ & 864,00 & 117 & 785,37 \\
\hline ITEM 31 & 2 & $5.150,00$ & 1841,89 & 391 & 5,57 \\
\hline ITEM 32 & 1 & 516,77 & 2360,79 & 206 & 8169,93 \\
\hline ITEM 33 & 2 & $236.084,00$ & 4252,72 & 109 & 1,35 \\
\hline ITEM 34 & 2 & $1.249,18$ & 6081,60 & 177 & 333,33 \\
\hline
\end{tabular}

Fonte: os autores. 


\subsection{Apresentação dos Resultados}

Para verificação da sensibilidade da disponibilidade diante da variação do TAT, foram gerados diversos resultados através da variação do turnaround time, desde 15 até 300 dias. A verificação é realizada na disponibilidade por suprimento, pois os dados considerados não incorporam as paradas por manutenção preventiva. $\mathrm{O}$ gráfico mostrado na figura 02 apresenta o comportamento através dessa variação, considerando o giro existente na FAB.

Figura 02 - Gráfico de disponibilidade e turnaround time considerando o giro existente na FAB.

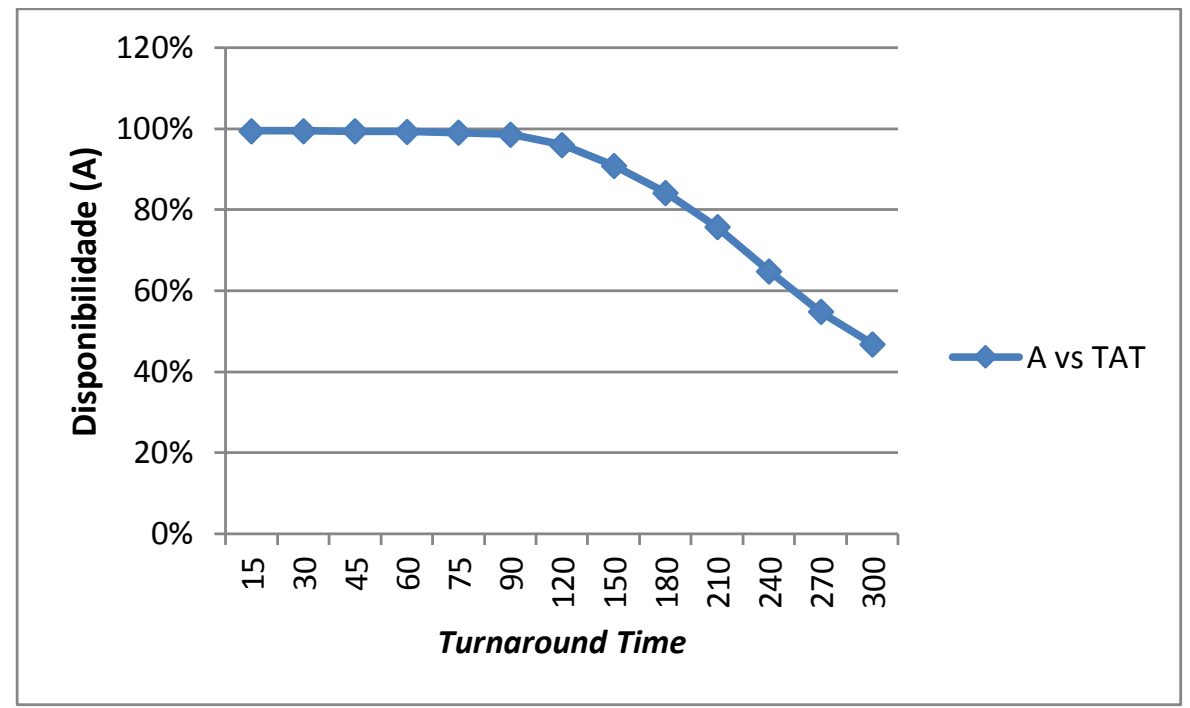

Fonte: os autores.

E ainda, foram aplicadas as mesmas variações de TAT para o caso de uma criação de uma Lista de Aprovisionamento Inicial (LAI) com o menor investimento possível, ou seja, sem giro. Nesse caso, o impacto do TAT se apresenta muito mais sensível até os 120 dias. A figura 03 mostra as duas curvas, para verificação de sensibilidade dos casos de consideração com o giro atual da FAB e sem giro. 
Figura 03 - Gráfico de disponibilidade e turnaround time considerando o impacto na disponibilidade com e sem giro existente na FAB.

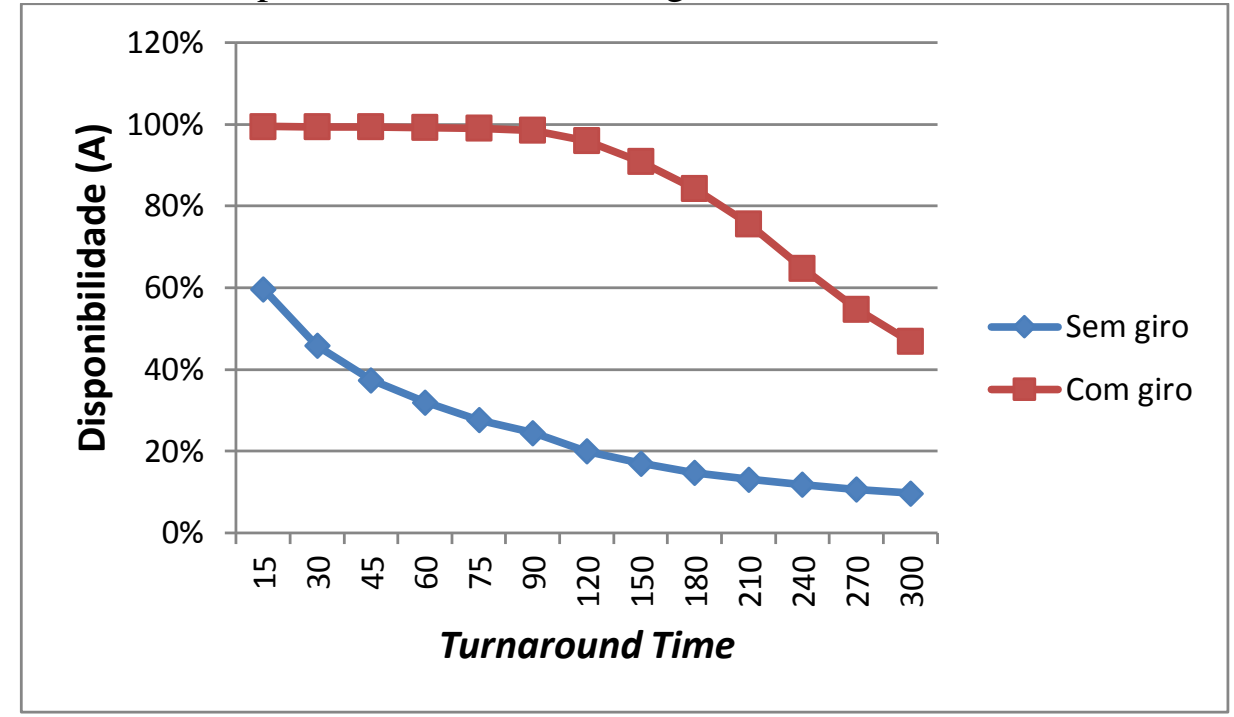

Fonte: os autores.

Em análise ainda da proposta do sistema configurado no OPUS, foi realizada uma verificação do investimento executado pela FAB para os itens listados e uma possível melhoria para o acervo atual encontrado, com o foco de aumento de disponibilidade. No gráfico da figura 04 é apresentado o ponto inicial do acervo que foi levantado na FAB, que leva a uma disponibilidade máxima de $63.66 \%$, com uma previsão de investimento e o comportamento da disponibilidade de acordo com o valor investido nos itens listados. Assim, com um investimento de pouco menos de um milhão de dólares, ou seja, aproximadamente $0.5 \%$ do valor do acervo, a disponibilidade seria acrescentada em aproximadamente $35 \%$, o que acarretaria em aproximadamente $100 \%$ da disponibilidade, considerando apenas os itens apresentados. Apesar de se tratar de uma disponibilidade de suprimento é importante destacar, nessa análise, a importância da utilização de softwares para previsão e planejamento de compra e contratação de serviços. 
Figura 04 - Gráfico de disponibilidade por custo variando-se o investimento no acervo dos itens reparáveis da frota C-95M da FAB.

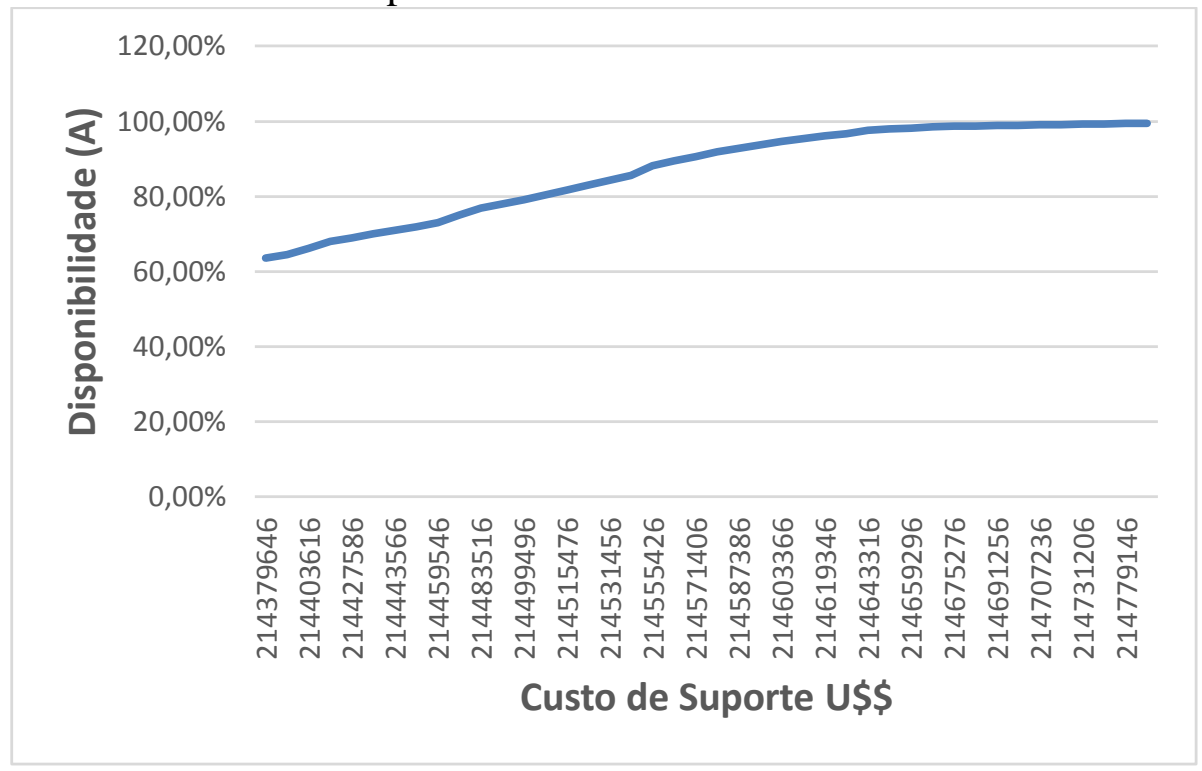

Fonte: os autores.

\section{Conclusões}

Através da análise da figura 02, é possível identificar que, com o giro atual dos itens, a partir de aproximadamente 120 dias de TAT, a disponibilidade diminui sensivelmente. Diante disto, para as características do sistema apresentado é possível criar um parâmetro para os novos contratos e requisições de componentes e serviços, de máximo tempo de reparo e entrega.

E ainda, como pôde ser visto na figura 03, a curva sem giro tem uma sensibilidade muito maior até os 120 dias de TAT, ou seja, seu impacto é limitado por outros fatores, como o giro. Considerando que a média ponderada do TAT, que considera a quantidade de recolhimentos de cada item estudado e o tempo de reparo, é de 210 dias, é possível verificar que a disponibilidade por suprimento máxima do projeto é de aproximadamente $78 \%$, figura 2 . A meta atual de disponibilidade operacional do projeto é de 50\%, mas nesse valor são incorporadas as paradas por manutenções programadas, além de todo o restante de itens das aeronaves que não estão inseridos neste estudo. No caso de análise desse TAT para o giro zerado da frota, figura 3, a disponibilidade não chega a $20 \%$, sendo que, se consideradas as manutenções preventivas, seria ainda menor.

Na verificação dos resultados através da aplicação do TAT real levantado pelos dados de ordens de serviços, de reparos realizados internamente e em empresas contratadas, a disponibilidade retornada pelo software foi de $63,66 \%$. Dessa forma, é possível identificar que 
os tempos médios de reparo e disponibilidade para o operador impactam significativamente na disponibilidade da aeronave e estão acima dos 120 dias estipulados como melhoria.

Em relação aos custos de investimento, o valor do acervo atual está acima de duzentos milhões e, utilizando o software para a análise de uma possível LAI, o valor do investimento, para a mesma disponibilidade (97\%), seria de pouco mais de vinte milhões, ou seja, cerca de 10\%, análise feita para um TAT de 120 dias. Diante do exposto, é possível verificar que é necessário implementar ferramentas de gerenciamento para melhorar o planejamento de investimentos na gestão de estoque e suprimento logístico da FAB.

\section{Considerações Finais}

A manutenção de diversos sistemas complexos, como são os da FAB, demonstram que a aplicação de metodologias de gerenciamento e softwares é necessária para a melhoria dos processos. Conforme verificado, o TAT é um parâmetro que impacta consideravelmente na disponibilidade da frota e, ainda, com o acervo bastante elevado encontrado, a sensibilidade deste parâmetro foi menor que quando considerada uma LAI (sem giro), até o valor de 120 dias de TAT. Além disto, evidenciou-se que softwares como o OPUS 10 podem auxiliar nas tomadas de decisão e contribuir para a melhoria dos processos de análise custo-eficiência.

Conforme citado em Tysseland (2009), quando o OPUS foi utilizado nos projetos de defesa na Noruega, ele teve sua aplicabilidade diminuída devido à falta de informações e, naqueles em que foi aplicado, a redução de custos foi de $60 \%$. O mesmo ocorre na FAB, quanto à falta de informações dos projetos; dessa maneira, é necessário o desenvolvimento das ferramentas de controle da frota.

Conforme demonstrado na pesquisa bibliográfica, a FAB está no mesmo caminho das outras organizações militares mundiais, quando utilizam o modelo de múltiplo escalão para atendimento logístico da frota.

A partir dos resultados, é possível identificar que, com o acervo apresentado, um parâmetro para utilização nos contratos e requisições, seja de serviço ou aquisição, é de 120 dias, pois, a partir desse prazo, o impacto na disponibilidade se torna mais acentuado e, com isso, menos atrativo para a FAB.

Como trabalho futuro, sugere-se que seja realizada uma separação entre as ordens de serviço atendidas internamente e externamente para verificação da viabilidade de terceirização dos serviços diante do impacto de prazo de atendimento e disponibilidade. E, ainda, é necessário 
um controle maior das informações de parâmetros como MTBFe TAT, para que o estudo possa ser aplicado para um maior número de equipamentos das aeronaves e até outros projetos.

Sugere-se também, que o controle do acervo e do giro sejam precisamente realizados e também que se incluam os tempos de parada para manutenção corretiva. Com esses dados, é possível disponibilizar informações mais precisas para que sejam tomadas as decisões de investimento e metas de desempenho. 


\section{Referências Bibliográficas}

FREITAS, Thiago P. Rodrigues de. Terceirização logística: um estudo sobre as vantagens e desvantagens de sua implementação. 2014.

GRAVES, S. C. A multi-echelon inventory model for a repairable item with one-for-one replenishment. Management Science, Cambridge, v. 31, p. 1247 a 1256, outubro de 1985. ISSN 10.

JR., V. D. R. G.; SRIVASTAVA, R. Repairable inventory theory: Models and applications. European Journal of operational reasearch, 1 de abril de 1997. 1-20.

KENEDDY, W. J.; PETTERSON, J.; FREDENDALL, L. D. An overview of recent literature on spare parts inventories. International Journal of Production Economics, 14 de junho de 2002. 201-215.

LAU, H. C.; SONG, H. Two-eschelon repairable item inventory system with limited repair capacity under nonstationary demands. Institutional knowledge at Singapore management university, p. 1901 - 1908, 2004.

MOTA, D. C. VARI-METRIC Assumptions Study: A Simulation Approach. Londres. 2014.

SHERBROOKE, C. C. Optimal inventory modeling of systems. 2. ed. Boston: Kluwer Academic Publishers, 2004.

SLEIDERINK, W. S. Reducing penalty costs in performance-based contracts. Enschede: [s.n.], 2015.

TYSSELAND, B. E. Spare Parts optimization process and results. International Journal of Physical, Distribution \$ Logistics Management, janeiro de 2009. 8-27.

WAAK, O.; STURGESS, P. Evaluating cost effectiveness and risk of third party support. Defense Logistics Support Conference. [S.l.]: [s.n.]. 2000.

WIJK, O.; ANDERSSON, P. Simulation as support for PBL Contract Design. Proceedings of eMaintenance 2012. Kulturens Hus: Lulea University of Tecnology. 2012. p. 37-42.

WU, M.-C.; HSU, Y.-K. Design of BOM configuration for reducing spare parts logistic costs. Expert System with Applications, Hsin-Chu, v. 34, n. 4, p. 2417-2423, maio de 2008. 


\title{
Analysis of the impact of repairable Turnaround Time on the availability on the C-95M Project fleet
}

\begin{abstract}
This paper analyzes the relationship between the average Turnaround Time (TAT) of repairables itens and the impact on the availability of the C-95M project fleet, using historical data from the Brazilian Air Force (FAB). Such an approach of paramount importance for the work carried out at the FAB, since the results may impact on the need for the increase of the spares, which entails larger amount of budget and/or a smaller quantity of aircraft ready for flight. The objective of this work indentify how much the TAT performance of the contracted companies impact the availability of the aircraft. This attempt will be achieved through a bibliographic review regarding the subject, data collection of the Brazilian Air Force reality and using OPUS 10 in the modeling step to verify results and indentification of improvements in the process. The analysis showed that TAT impacts the availability, occurring more intensely in scenarios with smaller amounts of spares. For stock existing from FAB C-95M project, it was possible to verify that the impact is bigger when the TAT exceeds 120 days, approximately. In this way it was possible to identify that this parameter is advantageous use in new acquisitions and hiring of services.
\end{abstract}

Keywords: TAT. Availability. C-95M. Brazilian Air Force. 